Check for updates

Cite this: RSC Adv., 2018, 8, 9223

Received 11th November 2017

Accepted 21st February 2018

DOI: $10.1039 / \mathrm{c} 7 \mathrm{ra12347g}$

rsc.li/rsc-advances

\section{Vancomycin-loaded titanium coatings with an interconnected micro-patterned structure for prophylaxis of infections: an in vivo study}

\author{
Hao Zhang, $\dagger^{a}$ Guangchao Wang, (D) $\dagger^{a}$ Peizhao Liu, $\dagger^{a}$ Dake Tong, ${ }^{a}$ Chen Ding, ${ }^{a}$ \\ Zequan Zhang, ${ }^{\mathrm{b}}$ Youtao Xie, ${ }^{\mathrm{b}}$ Hao Tang*a and Fang $\mathrm{Ji}^{\star a}$
}

\begin{abstract}
Titanium (Ti) and its alloys are widely applied as orthopedic implants for hip and knee prostheses, fixation, and dental implants. However, $\mathrm{Ti}$ and its alloys are bioinert and susceptible to bacteria and biofilm formation. Thus, surface biofunctionalisation of $\mathrm{Ti}$ is essential for improving the biofunction of Ti. The current in vitro study indicated that calcium phosphate bone cement with vancomycin doped on micropatterned $\mathrm{Ti}$ with a grid-like structure surface could preserve the property of inhibition of bacterial adhesion and biofilm formation while not affecting the osteogenic differentiation. The present study investigated whether the biological performance of the bactericidal effect is preserved in vivo. The rabbit osteomyelitis model with tibial medullary cavity placement of $\mathrm{Ti}$ rods was employed to analyze the antibacterial effect of vancomycin-loaded Ti coatings with interconnected micro-patterned structure (TV). Thirty female rabbits $(N=10)$ were used to establish the implant-associated infection. Prior to implanting the TO and TV rods into the medullary cavity of the left tibia of the rabbits, $10^{6} \mathrm{CFU} \mathrm{mL}^{-1}$ methicillin-resistant Staphylococcus aureus (MRSA) was injected into the medullary cavity of the left tibia of the rabbits. The sterile Ti rod (NT) was used as the blank control. After 3 weeks, bone pathology was evaluated using $\mathrm{X}$-ray and micro-CT. The in vivo study proposed that TV has the potential for prophylaxis against MRSA infection. Thus, the interconnected micro-patterned structured Ti rods loaded with vancomycin could be applied for preventing Ti implant-associated infections.
\end{abstract}

\section{Introduction}

Titanium (Ti) and its alloys are widely used in orthopedic implants such as hip and knee prostheses, fixation, and dental implants. The primary causes of failure of orthopedic implants are aseptic loosening and infection. ${ }^{1}$ An orthopedic prosthesis, once implanted, may be affected by microbial infection, especially following open fracture fixation. ${ }^{2,3}$ The ultimate goal of fabricating orthopedic Ti implants should be to avoid complications post-implantation. Fabricating an antibacterial $\mathrm{Ti}$ implant would facilitate the prophylaxis against Ti implantassociated infection.

A leading cause of Ti implant-associated infection is that Ti is bio-inert, making it easy for the bacteria to adhere to the surface and form biofilms. Thus, Ti implant-associated infection could be prevented by inhibiting the bacterial adhesion and biofilm formation by applying immobilized antibacterial

\footnotetext{
a Department of Orthopedics, Changhai Hospital, The Second Military Medical University, No. 168 Changhai Road, 200433, Shanghai, China. E-mail: doctorjif@ 126.com; tanghao1978@163.com; Tel: +86-021-31161696; +86-021-31161682

${ }^{b}$ Key Laboratory of Inorganic Coating Materials, Shanghai Institute of Ceramics, Chinese Academy of Sciences, Shanghai, China

$\dagger$ These authors contributed equally to this work.
}

agents to the Ti surface. Another cause of infection is that the host factors for the in vivo Ti implant are present in a relatively immunosuppressive zone, and even a small number of bacteria can lead to severe $\mathrm{Ti}$ implant-associated infection. ${ }^{4}$ The $\mathrm{Ti}$ surface is susceptible to bacterial adherence and biofilm formation; and thus, surface modification of Ti is a fundamental approach for the fabrication of antibacterial $\mathrm{Ti}$ implants. In the past decade, biofunctionalisation of Ti with antibacterial properties has mainly focused on the immobilization of antibacterial agents, including antibiotics, antimicrobial peptides (AMPs), and inorganic ions, to the Ti surface..$^{5-9}$ It divided into two parts namely passive or active antibacterial Ti surface. The passive antibacterial Ti means these Ti surface can inhibit the bacteria adhesion or kill the bacteria in contact. For example covalent bond vancomycin on the Ti surface. ${ }^{\mathbf{1 0}}$ While the active antibacterial Ti surface means this Ti surface can locally deliver antibacterial agents and kill the bacteria around the Ti implant, for example, fabricate titanium nanotube on the titanium surface and loading vancomycin to improve the antibacterial activity or fabricated vancomycin coated titanium with electrospinning nanotechnique to treat implant associated infection with control release of vancomycin. ${ }^{11,12}$ Biofunctionalised Ti can either kill the bacteria upon contact or release antibacterial agents locally. However, 
disadvantages such as cytotoxicity of the antibacterial agents, a local burst release of the antibacterial agents, and bacterial resistance are also exhibited by this method.

Bone tissue is a hierarchical composite structure, sized from $\mu \mathrm{m}$ to $\mathrm{nm}$; the structure of each level is rather micro and orderly at the next lower level structure. Haversian osteon is the basic building blocks of the normal bone structure of the human body; such a tubular structure in approximately $150-250 \mu \mathrm{m}$ in diameter. These structures arranged in concentric rings are interweaved, connected to each other within the lacuna that is filled with tissue fluid, intertwined with the porosity of $19 \%$ of the tubular structure, and medullary cavity material with adequate blood circulation, exert the effect of transport corridor. The morphology of bone tissue micron scale on the surface of the bone tissue determines whether the existing implant material achieves priority as compared to the coarse porous surface. If some antibiotics are loaded, and the release of the antibiotics on the surface of the porous implants can be controlled, the performance of antibacterial effect can be further improved.

The implant surface micro-topography influences the bioactivities, such as anti-inflammatory and anti-fibrotic properties, of the biomaterials. ${ }^{\mathbf{1 3}}$ For example, Ti with surfaceoptimized nano-micro-hierarchical structure exhibits topography-dependent antibacterial capacity as it can inhibit bacterial adhesion at the early stage and provide better osteogenesis ability than the micro-scaled surface. ${ }^{\mathbf{1 4 , 1 5}}$ The current in vitro studies showed that the micro-pattern structures of titanium coating loaded with vancomycin strongly inhibits bacterial adhesion and biofilm formation. Compared to an ordinary Ti coating through the hole structure, loading with vancomycin through the hole pattern structures between the Ti coating is beneficial to bone marrow mesenchymal stem cell adhesion, proliferation, and osteogenic differentiation; the small local doses of the drug do not negatively impact the cell behavior. ${ }^{\mathbf{1 6}}$ The animal research was based on the in vitro experimental studies that assessed the loading with vancomycin through hole pattern structures of Ti coating of anti-infection effect in the body. Thus, we used methicillin-resistant Staphylococcus aureus (MRSA) bacterial strains for the simulation of acute bacterial infections and implant materials in the proximal tibia intramedullary implant bacteria consecutively. The follow-up evaluation of the vancomycin load through the hole pattern structure of the Ti coating gave rise to a local anti-infection effect. In addition, we also observed the state of infection on the antibacterial coating plant-bone interface with respect to bone integration ability.

\section{Material and methods}

\section{Materials}

Ti6AL4V rod $(2.0 \times 20 \mathrm{~mm}, 2.0 \mathrm{~mm}$ in diameter, and $20 \mathrm{~mm}$ in length) was used, vancomycin (Sigma-Aldrich, USA), MRSA ( $S$. aureus, ATCC 43300). All experiments were performed in compliance with the relevant laws or guidelines, following the institutional guidelines.

\section{Methods}

Preparation of Ti rods with micro-patterned structure. Ti coating spray (pure Ti coating), plasma spray with the placeholder filler metal etching method, and spraying preparation solution (with a through hole design Ti coating) were utilized. The cutting machine cut the Ti rod at a length of $20 \mathrm{~mm}$ to form Ti-coated sample rod that was washed with anhydrous alcohol and distilled water ultrasonically. This approach was in accordance with the method of preparation of conventional titanium coating (T0) and vancomycin-loaded in the Ti through-hole design of the titanium coating as per previous study (TV). ${ }^{\mathbf{1 3}}$ The structure diagram and scanning electron microscopy (SEM) were shown in Fig. 1.

Experiment group and bacterial culture. The in vivo experiment was divided into three groups: pure Ti coating group (T0), micro-pattern Ti coating loaded with vancomycin (TV), and the sterile Ti rod (NT) was used as the blank control. Standard strains of MRSA, (ATCC, USA) were used in this experiment. The cultivation is the second part of the preparation of the NT group, finally the sterilized trypticase soy broth (TSB) were used

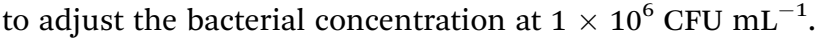

In vivo experiment. All in vivo animal experimental procedures were approved and performed according to the guidelines of the Animal Ethics Committee of Shanghai Changhai Hospital, China. Thirty female rabbits $(2-2.5 \mathrm{~kg})$ were used. The rabbits were divided into three groups. The T0 and TV rod samples were

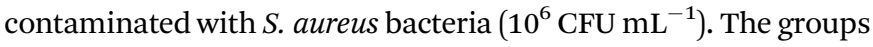
were denoted as T0 + MRSA, TV + MRSA, and T0 + PBS (negative control). Then, the Ti rod in the three groups $(N=10)$ was implanted into the left tibia of the rabbits via the tibia plateau. Briefly, the knee of the rabbits was opened to expose the plateau (similar to the entry point of the tibial intramedullary nailing), and the bone cavity was expanded with electroporation until it was sufficiently large to hold the Ti rod. After implanting the Ti rods, the surgical site was closed layer-by-layer, following the opening of the hole in the tibia plateau that was sealed with bone wax. The rabbits were maintained in separate cages and allowed food and water ad libitum.

All animals should not be used antibiotics to prevent infection postoperatively. Each rabbit cage was maintained at a constant temperature under observation for 3 days, followed by transfer to the ordinary single cage breeding room. After 42 days, the experimental animals are executed by embolism, under sterile conditions. The right side of the tibia was examined in different groups of animals, respectively.

The observations were recorded at the beginning of the operation, followed by that in the first week, then at 2, 4, 5, and 6 weeks after surgery. The record contents include the weight of animals by electronic balance and digital infrared thermometer body (Terumo, Zhejiang, China) for measuring the ear temperature. Furthermore, the position of the rabbit ear margin vein is marked to withdrawn an optimal volume of the venous blood sample at different time points. Leukocytes serve as technical indicators for a routine blood test. At different observation time points, the right side of the rabbit knee joint 

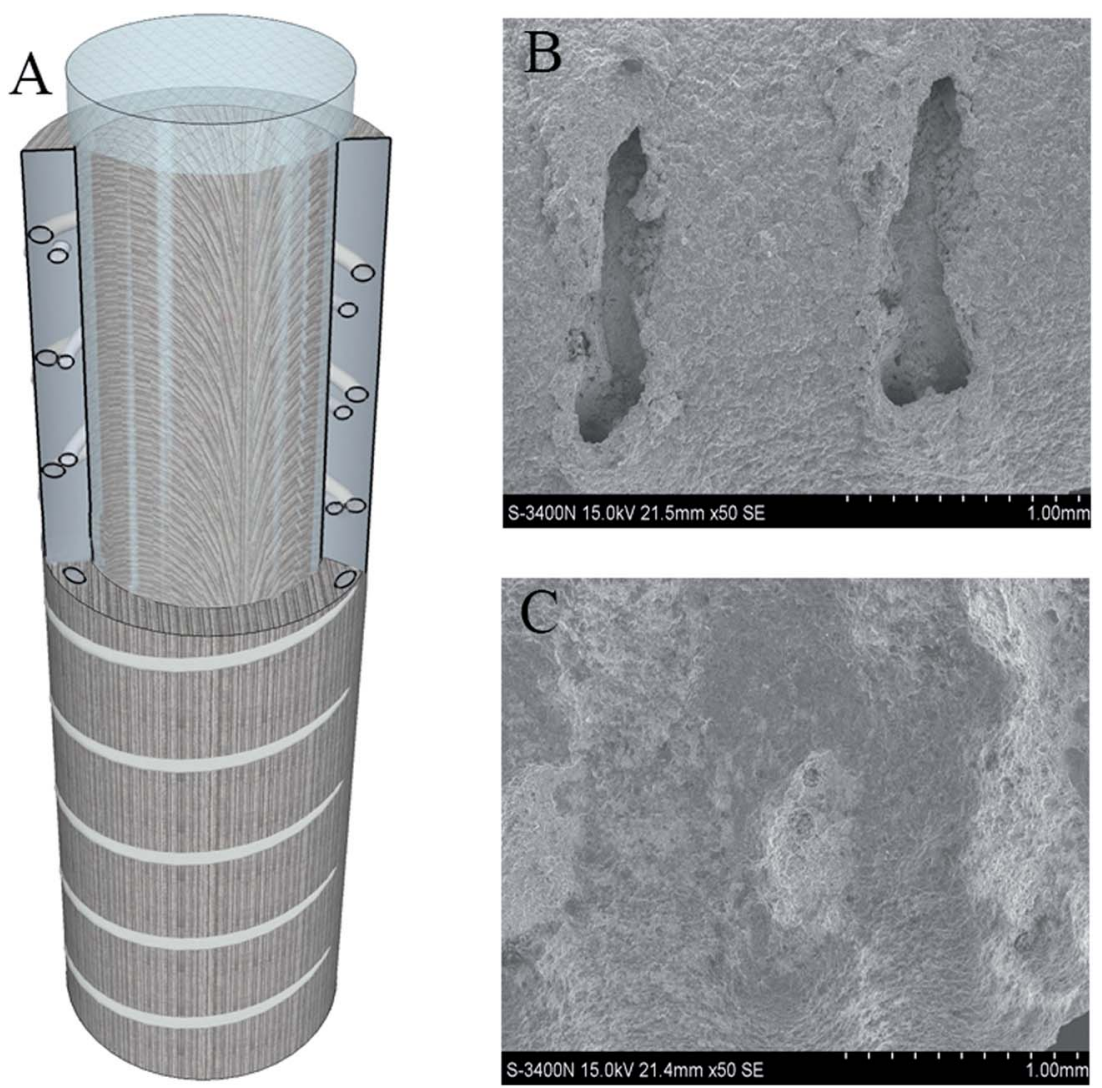

Fig. 1 Ti rod surface coated with a Ti coating through a hole-structure pattern: (A) structure diagram; (B) SEM before drug loading; (C) SEM after drug loading.

swelling profile, the presence of seepage at the surgical incision site, and exudate traits were recorded.

\section{Ti implant-associated infection characterization}

General observation. At 6 weeks, all groups of experimental animals were sacrificed using the air embolism method; 3 animals were randomly selected and tibia removed under aseptic conditions. The sagittal longitudinal was cut open to obtain the specimens as described previously. ${ }^{17,18}$

SEM characterization. Each of the above implants and bone samples was washed three times with PBS, fixed with $2.5 \%$ glutaraldehyde solution, dehydrated gradually using 50, 70, 80, 90 , and anhydrous alcohol, followed by dehydration for $10 \mathrm{~min}$ and air-drying.

Analysis of bacteria in the peri-implant. 6 weeks after surgery, the animals were sacrificed under aseptic conditions to remove the column specimens of the tibia with the coating. The coating under aseptic conditions on the column is obtained from the tibia and cultured on the pancreatic soy peptone agar plate (Trypticase soy agar, TSA), followed by incubation at $37^{\circ} \mathrm{C}$ for $24 \mathrm{~h}$. Thus, the coated board after each TSA training board was examined for the bacterial growth and colony morphology.

Another sample of embedded column coating material was collected by ultrasonic oscillation, diluted 10 -fold, and cultured similar to the TSA coating plate. The proliferation of the bacteria was enumerated quantitatively.
Three tibiae were randomly selected from each group, weighed, and conserved in liquid nitrogen. Then, the specimens were crushed and ground into bone samples (all processed were conducted under aseptic conditions). The bone powder was transferred to a $15 \mathrm{~mL}$ centrifuge tube containing $6 \mathrm{~mL}$ PBS and centrifuged at $10000 \mathrm{rpm}$ for $10 \mathrm{~s}$ (Vortex, Germany). Subsequently, $100 \mu \mathrm{L}$ of the supernatant was serially diluted and plated on agar plates for overnight culture at $37^{\circ} \mathrm{C}$. The bacteria colonies were counted, and the viable bacteria count was calculated; the $\mathrm{CFU} \mathrm{g}^{-1}$ bone was calculated.

Histopathological analysis. After six weeks, the rabbits were sacrificed by air embolism. Any three specimens, from the three groups, were selected from the upper tibia. Subsequently, the tibiae were fixed with $4 \%$ paraformaldehyde at room temperature for $24 \mathrm{~h}$ and decalcified for 3 weeks using a rapid decalcifier (DeCa DX-1000, Hong Kong). Then, the tissue was fixed with $4 \%$ paraformaldehyde, embedded in paraffin, sliced, and subjected to HE staining, followed by visualization using optical microscopy to observe bone absorption around the bone tissue.

Micro-CT analysis of the upper tibia. The rabbit tibiae were fixed with $4 \%$ paraformaldehyde solution at room temperature for $24 \mathrm{~h}$ and rinsed in the water overnight. Three tibiae were analyzed using micro-CT by $3 \mathrm{D}$ reconstruction and quantitation. The scanning resolution was set at $20 \mu \mathrm{m}$ to estimate the bone trabecular thickness and the separation degree trabecular bone. 
In vivo cytotoxicity. The rabbits were sacrificed after 6 weeks. The heart, liver, spleen, and kidney samples were harvested, sliced, and stained with HE to observe cell toxicity in the gut tissues of the experimental animals.

Statistical analysis. The data were obtained from three repetitions of each experiment and analyzed by SPSS 19.0. The quantitative data were expressed as mean \pm standard deviation. The between-group comparison was performed by single factor analysis of variance (ANOVA), and multiple groups using the Student-Newman-Keuls test (SNK) test. $P<0.05$ indicates statistically significant difference.

\section{Results}

\section{General observation}

No animal death occurred during the surgery and experimental duration, and none of the rabbits showed any obvious systemic complications. All the rabbits in the T0 group showed knee joint swelling, suppuration, and local infection symptoms; with the passage of time, the local infection degree aggravated gradually; however, the rabbits in the NT and TV groups did not show any obvious signs of local infection. The average body weight of all animals decreased gradually from days 1-7 and increased from days 7-42. The weight of rabbits in the TV group was stable and increased during the observation period. The mean body weight of the T0 group was significantly lower than that of the TV group $(P<0.05)$. At day 42 , the mean body weights of the T0, TV, and NT groups were $2.15 \pm 0.20,2.56 \pm 0.07$, and $2.43 \pm 0.18 \mathrm{~kg}$, respectively (Fig. 2A). The average ear temperature of the three groups of rabbits increased gradually from day 1-5 after the operation. However, the TV group had a lower body temperature than the other groups. From day 5 to week 6 , the body temperature of the T0 group was higher than that of the TV and NT groups $(P<0.05)$, which might be attributed to the tibial intramedullary infection $(P>0.05)$ (Fig. 2B). No significant difference was observed between the TV and NT groups $(P>$ 0.05). The white blood cell count of the TV and NT groups were lower than those of the other groups $(P<0.05)$ (Fig. $2 \mathrm{C})$, which was in agreement with the results of the body temperature observation. The white blood cell count in the three groups showed a rapid increase in the first 3 days post-surgery; however, the white blood cell count in the TV group was lower than that in the other groups. The white blood cell count of the TV and NT groups decreased to normal after 1 week, while the T0 group continued to remain higher than the normal during observation period, and showed a significant difference as
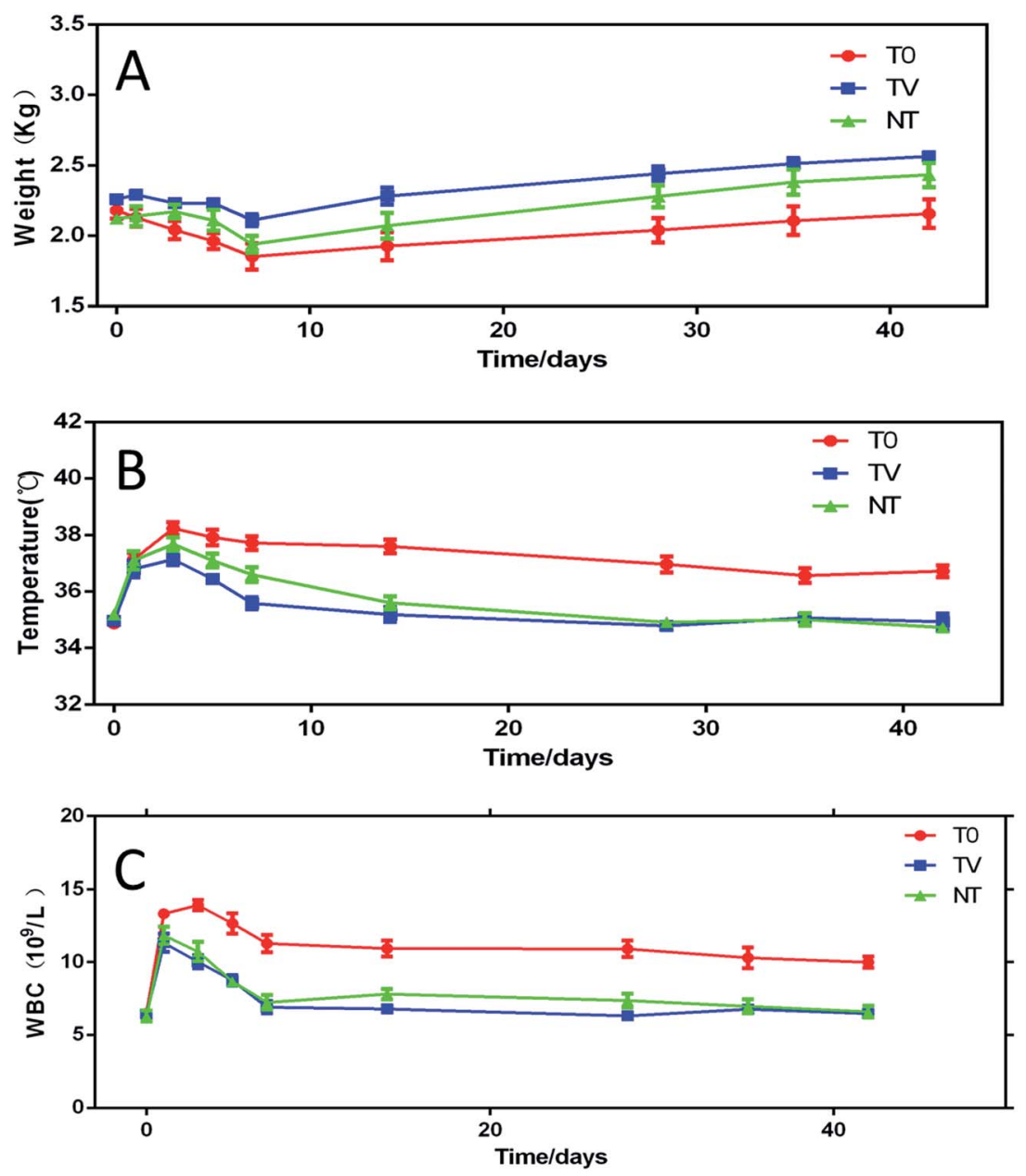

Fig. 2 Changes in the experimental animals during the observation period: (A) body weight; (B) body temperature; (C) white blood cell count. 

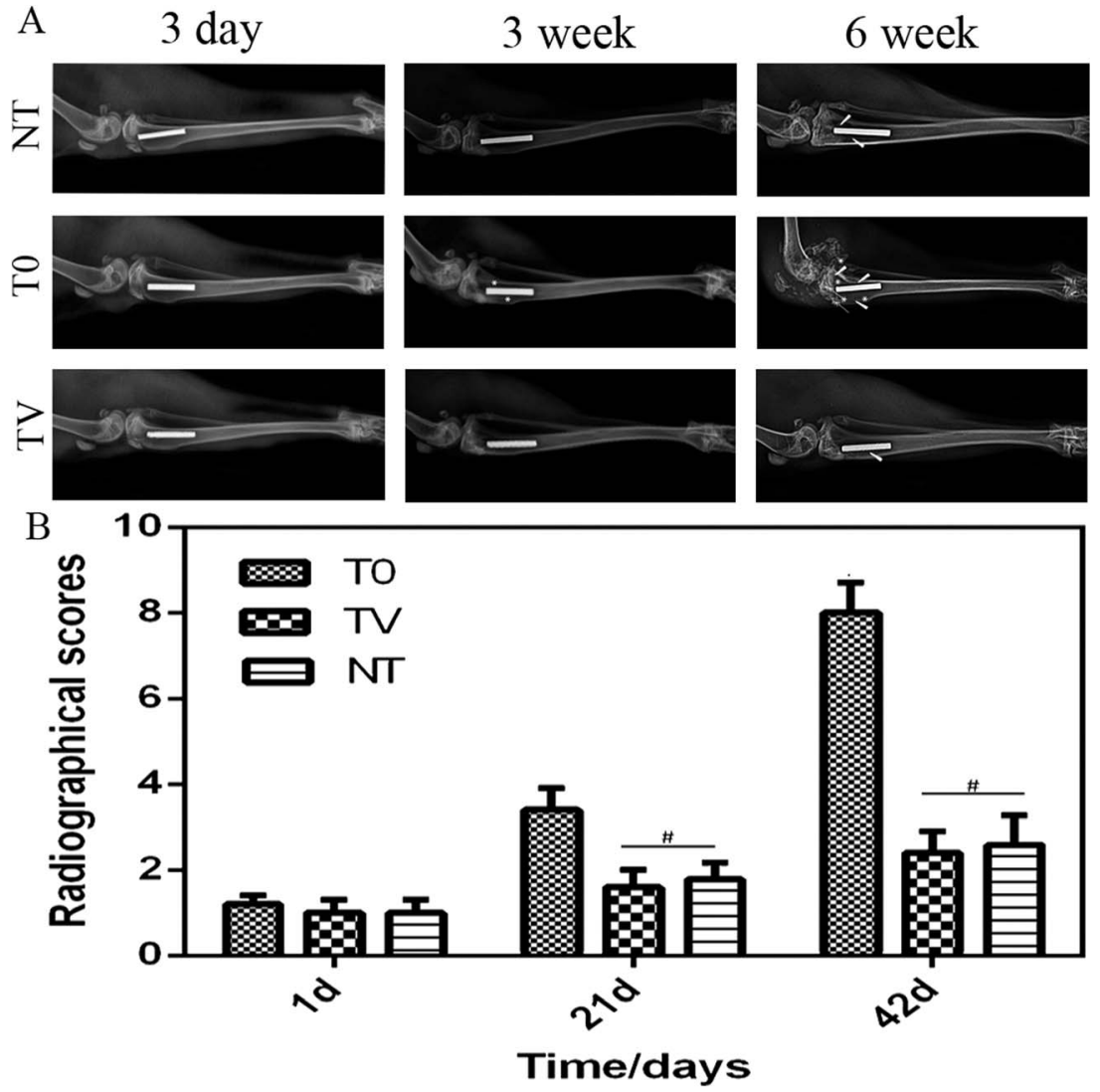

Fig. 3 Three groups of postoperative X-ray image scoring: (A) X-ray image of each group after surgery; (B) radiographical scores of the T0, TV, and NT group. On the first day after operation, no significant difference was observed between the three groups $(p<0.05)$. The scores of T0 group were significantly higher than those of the TV and NT groups $(p<0.05)$ no significant difference was observed between the animal scores (\#p > 0.05).

compared to the TV and NT groups (Fig. 3C), which was consistent with the results of body temperature observation.

\section{Radiographical evaluation}

Fig. 3 demonstrated that 1 day after Ti implantation at the bone, no significant changes were observed in the local bone, and the $\mathrm{Ti}$ and the bone combined closely. At 3 weeks after the operation, a mild bone resorption was noted around the implant, and some bone tissue was absorbed. A periosteal reaction was also observed; however, no significant changes were observed in the TV and NT groups. At 6 weeks after the operation, osteolysis and absorption occurred in the T0 group. No slight difference was observed between the TV and NT groups (Fig. 3). The scores of $\mathrm{X}$-ray radiographs in the three groups were quantified as shown in Fig. 3. The scores of the TV and NT groups showed no significant difference $(P>0.05)$; however, the T0 score was significantly higher in the TV group $(P<0.05)$. At 6 weeks, the mean scores of T0, TV, and NT were $8 \pm 1.41,2.4 \pm 1.02$, and 2.6 \pm 1.36 , respectively (Fig. 3).

\section{General observation of the rabbit tibia}

An abnormal accumulation of pus in the joint and medullary cavities, bone osteolysis, periosteal reaction, and bone structure of different performances were observed in the To group, which indicated the symptoms of infection, while the TV and NT groups did not show an significant change (Fig. 3). The quantitative analysis results showed that the standards of each rating scores were T0: $3.2 \pm 0.75$, TV: $0.60 \pm 0.50$, NT: $0.80 \pm 0.40$. No significant difference was observed between the TV and NT groups $(P>0.05)$. Compared to the T0 group, the average score of the TV group was reduced significantly $(P<0.05)$.

\section{Microbiological analysis of the implant and around the implant}

Ti implants from each group were harvested, and the bacteria in the Ti were counted with the tissue spread method. The results showed that abundant bacteria colony in the To group that encompassed the whole culture plate, as compared to the TV group. The growth in the TV group was lower than that in the T0 groups. The coated board in the NT group did not present an obvious bacterial growth (Fig. 4). The results of the quantitative analysis showed that the bacterial count in the T0 group reached as high as $8.42 \pm 0.68 \times 10^{5}$ CFUs, while it was $4.04 \pm$ $0.89 \times 10^{4}$ CFUs in the TV group (Fig. 4). The bacteria around the implant were also characterized as shown in Fig. 4. The electron microscopy results revealed that abundant bacteria covered on the implant surface, while only a small number of bacteria were observed in the bone tissue. The quantitative 

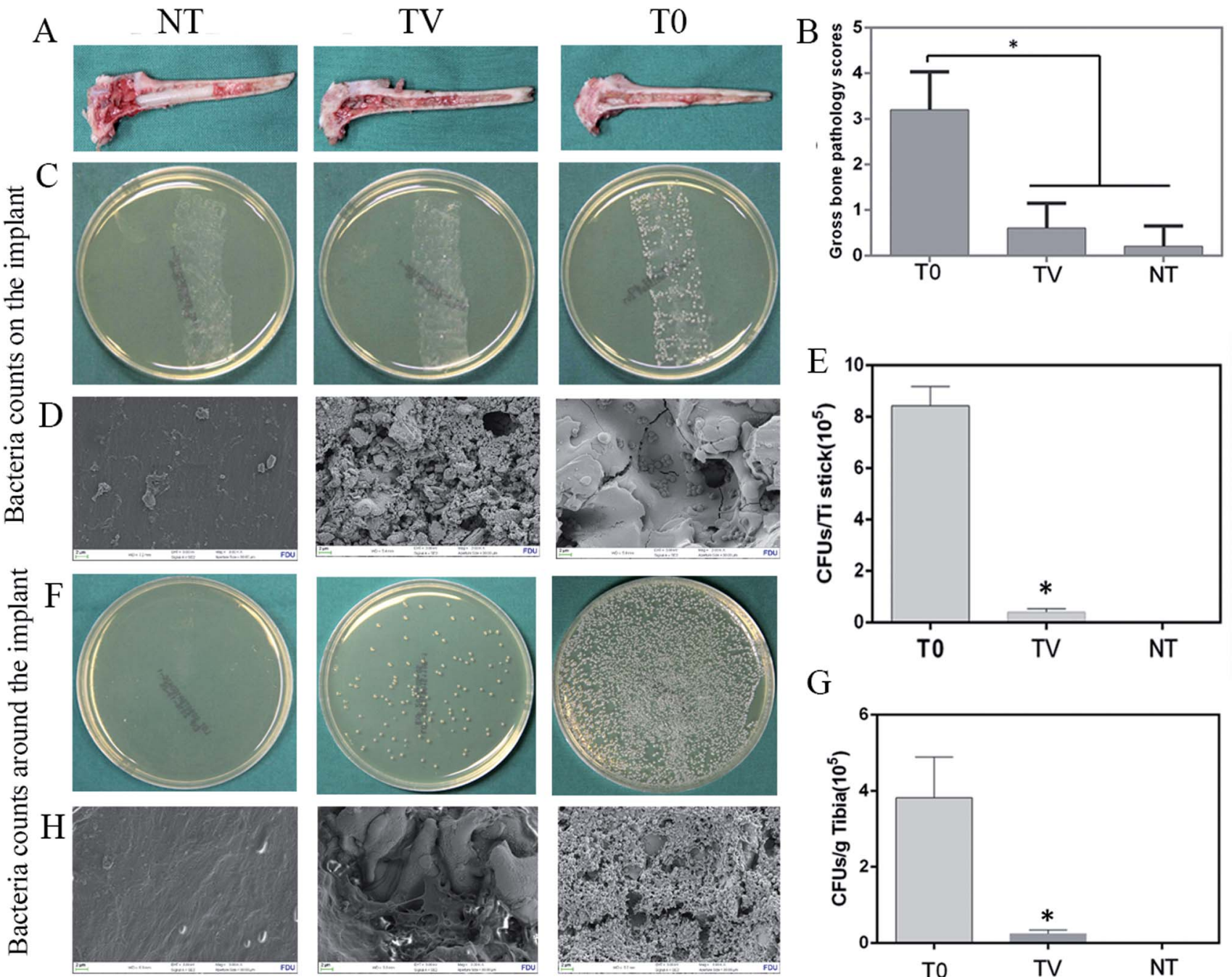

G

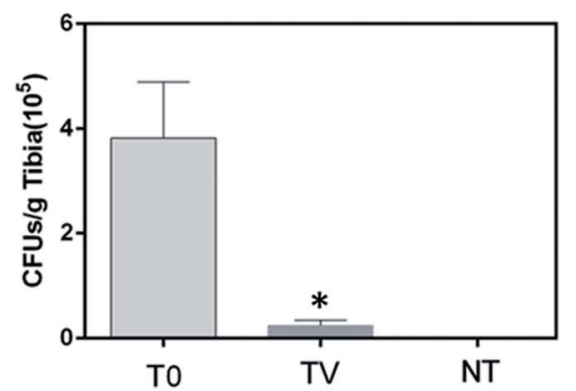

Fig. 4 Bacterial culture and quantitative analysis of Ti-coated column implants in three groups at 6 weeks: (A) gross appearance (B) gross score (C) TSA plate culture results; (D) SEM to characterize the bacteria on various Ti implants; (E) quantitative results of adherent bacteria on various Ti implants; (F) TSA plate culture results of the bacteria of the bone tissue around the implant; (G) quantitation of bacteria per gram of rabbit tibial bone tissue; (H) SEM to characterize the bacteria on various bone tissues around the Ti implant. TV and T0 were significantly different $(* p<0.05$ ); blank control group without bacterial growth.

analysis revealed that bacterial counts in the T0 group reached as high as $3.24 \pm 0.38 \times 10^{4}$ CFUs, while it was $3.04 \pm 0.37 \times$ $10^{3}$ CFUs in TV group (Fig. 4).

\section{Histopathological analysis}

HE staining showed the histopathology of the experimental rabbit tibiae in the transverse and longitudinal section. The T0 group of animals of the proximal tibia exhibit a decrease in the cortical destruction of bone trabecula, while the medullary cavity was infiltrated with a large number of inflammatory cells. The bone destruction was not observed clearly in the TV group, and the medullary cavity exhibited infiltration of a small number of inflammatory cells (Fig. 5). The NT group did not exhibit a distinct infiltration of inflammatory cells and bone structure, while the continuity of the bone cortex of remains intact and exhibits little inflammatory pathological changes (Fig. 5).

\section{Micro-CT analysis of the interface of the bone implant}

As shown in Fig. 6, the bone mineral density suffered severe damage, as evident from a large number of holes, while the bone tissue in TV and NT groups did not show any bone infection signs and the cortical bone remained intact. The quantitative data indicated that the bone tissue around the $\mathrm{Ti}$ implant suffered severe destruction due to MRSA infection as the bone tissue around the T0 was $8.74 \pm 1.67 \%$, which was much lower than the normal condition $(17.89 \pm 2.86 \%)$. Since the infection can destroy the plants surrounding normal tissue, the implants of bone and bone interface integration could also be affected. The bone tissue of the TV group remained intact, and the bone volume was $16.6 \pm 2.63 \%$, which showed no significant difference as compared to the NT group $(P>0.05)$. These results indicated that TV showed excellent antibacterial property in vivo. 
T0
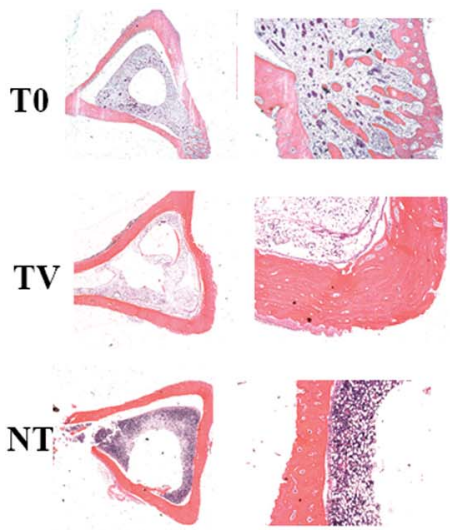
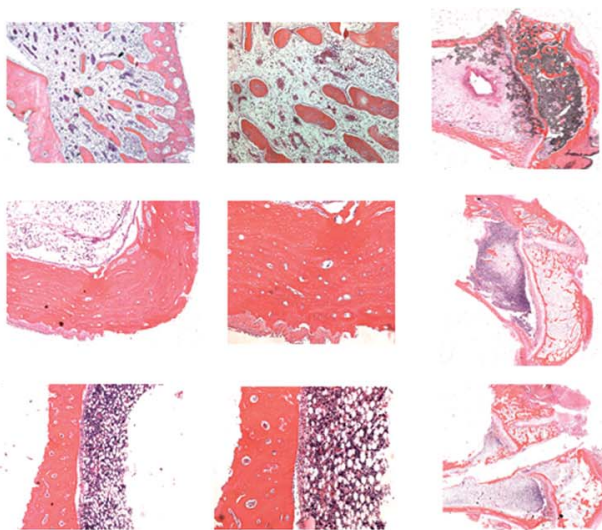
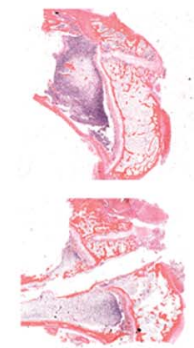
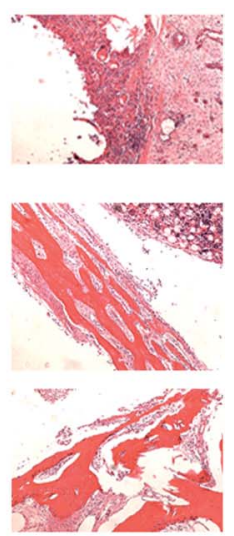

Fig. 5 Rabbit tibia cross-section HE staining. Column (I): no amplification; Column (II) ( $\times 50$ amplification): T0 group showed moderate-tosevere inflammation performance, TV group showed mild inflammatory response, NT group showed no obvious pathological changes in infection. Column (III) ( $\times 100$ amplification): T0 group indicates a large number of inflammatory cells, local visible bone destruction, trabecular bone reduction. Only a small number of inflammatory cells were seen in the TV group.

A
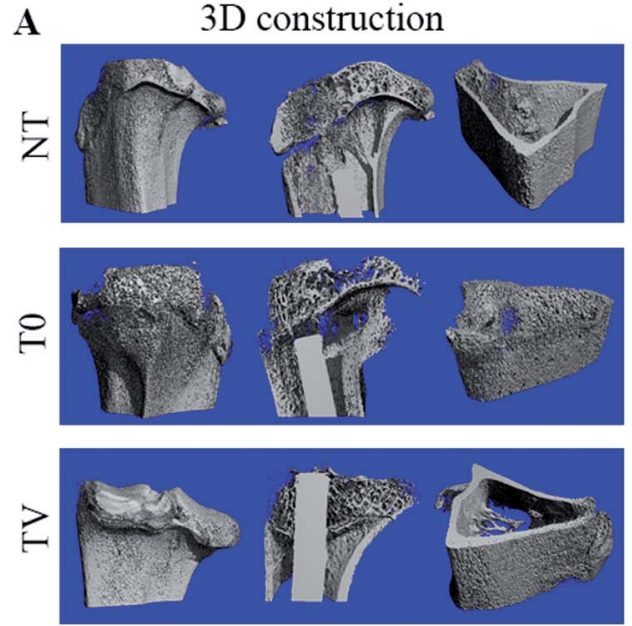

B

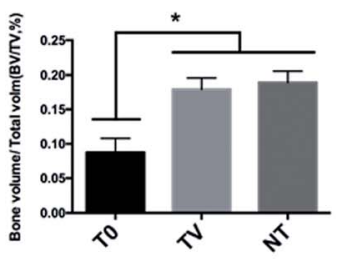

C

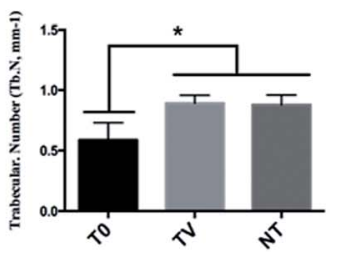

Fig. 6 Four measurement parameters for trabecular bone: (A) 3D reconstruction of the bone within implant; (B) quantitative results of BV/TV obtained from the micro-CT analysis; (C) quantitative results of Tb. $\mathrm{N}$ obtained from the micro-CT analysis. ${ }^{*} P<0.05$ comparing TO with TV and NT. BV/TV denote bone volume/total volume, Tb. N denote trabecular number.

\section{In vivo toxicity}

To investigate the toxicity of TV after release from the animal body, the influence of each group was studied by observing the liver histology of 3 cases (Fig. 7). The TV set of liver HE staining results did not detect any obvious pathological changes in the heart, liver, spleen, kidney tissues of the TV experimental animals.

\section{Discussion}

Owing to the extensive use of botanical materials in the field of orthopedics and the extensive application of broad-spectrum antibiotics, bacterial resistant strains appear sequentially, such as MRSA braising from acute chronic osteomyelitis and peripheral infection, which is continually increasing. ${ }^{19-21} \mathrm{~A}$ large number of resistant infections are usually difficult to cure resulting in prolonged treatment cycles. This characteristic might be attributed to the formation of biofilm on the bone and implant surface. ${ }^{22}$ Subsequently, the biofilm exerted a role of the protective umbrella, under which, the bacteria continued to proliferate and spread; however, the adamant drug resistance continues to resist the antibiotics and immune cells in order to phagocytose the pathogens. ${ }^{23,24}$ Therefore, the emergence of similar implant-related infection will have catastrophic consequences clinically. As a result, the doctor must remove the implant by surgery, such that the infection can be controlled effectively. This process requires not only huge medical resources but also the need for patients to bear a huge financial burden as well as, the patient will suffer extraordinary pain and pressure.

The most common approach to clinical prevention and treatment of plant-related infections is the loading of vancomycin-containing PMMA bone cement filled with infectious bone defects or removal of implants remaining within the 

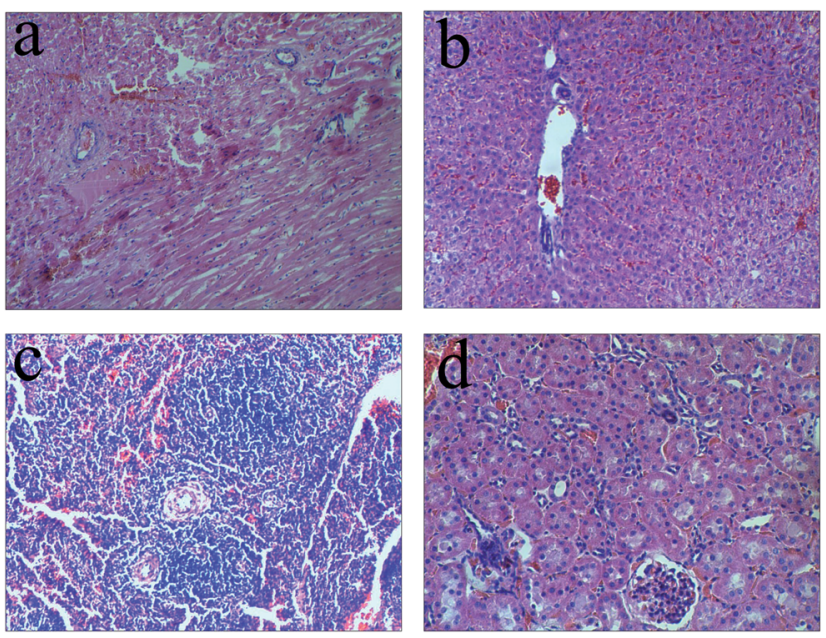

Fig. 7 Animals visceral HE staining of the TV group: (a) heart; (b) liver; (c) spleen; (d) kidney. No obvious pathological change was observed in visceral $\mathrm{HE}$ staining.

cavity for a local anti-infective effect. However, PMMA bone cement loaded with vancomycin could not degrade; this approach often requires repeated surgery for an efficient treatment. ${ }^{25}$ Our in vitro study demonstrated that vancomycin loaded through micro-patterned Ti coatings exhibit good antibacterial activity and have no significant effect on bone osteointegration around the implant. Next, we tested the anti-infective effect of the coating by additional animal experiments. In this study, the animal experiment was used to simulate the time operation of the tibial intramedullary nail. The MRSA pathogens were injected into the borehole of the proximal tibia to induce the intraoperative or postoperative bacterial contamination. The animal model is similar to the clinical infection of the implantassociated infection as compared to the clinical infection; the experimental condition is artificially produced bacterial contamination and causes acute bacterial infection.

Based on the general observation, imaging monitoring, microbiological analysis, and histopathological analysis for the evaluation of the implant-associated infection. The results showed that the body weight of the TV group continued to increase during the observation period and the ear temperature and venous blood WBC fluctuated slightly in the early observation period, and then gradually returned to normal with the passage of time. The T0 group showed the gradual emergence of the periosteal reaction around the implant, bone resorption, and the formation of sequestrum, which provides CT, gross specimens, and histopathological analysis results. The TV group coated with vancomycin effectively inhibited the development of peripheral infection in the proximal tibia of the rabbits, and the histological study confirmed that the localized antibiotics did not exert a distinct biotoxicity to the body.

Surgery is a damage of the local organization of the defense function, blood changes, hematoma formation, and necrotic tissue absorption; these factors could act as accelerators of a local bacterial infection. ${ }^{26}$ The systemic use of antibiotics for the prevention of such infection is also limited. Notably, the implantation of the plant in the specific operation occurs, even if the surgical incision area of the pathogens through different channels and planting; moreover, the infection occurrences is also much lower than the experimental rabbit planted with bacterial concentration. Therefore, the local application of vancomycin-coated $\mathrm{Ti}$ implants, with sustained release, is sufficient to kill a small number of invasive bacteria in the body as the release of antibiotics dose is limited. Different from other study that loading vancomycin through fabricating titanium nanotube on the titanium surface or with electrospinning nanotechnique. ${ }^{\mathbf{1 1 2} 12}$ Our study fabricated a micro-patterned structure on titanium for loading of vancomycin, which can also control release vancomycin in vivo and prevent implant associated infection.

Thus, we can speculate that the loading of vancomycin with a micro-patterned structure of Ti coating could kill the invasive pathogens through the local release of antibiotics; these antibiotics play a role in the prevention of infection. The purposes of the prevention of infection around the plant are expected to be achieved with such modified coating. For an ideal implant, despite the precaution of implant-associated infection, excellent bone implant osteointegration should be preserved. The loading of vancomycin with micro-patterned structure $\mathrm{Ti}$ coating surface morphology as well as the carrier of vancomycin calcium phosphate bone cement could promote osteogenesis for bone integration. In this part of the experiment, it was confirmed that the bone around the implant was not affected by MRSA. The osteointegration of the coating was substantiated by the in vitro stem cell experiment, and further investigation into in vivo bone integration was essential.

\section{Conclusion}

In vivo results showed that compared to the conventional $\mathrm{Ti}$ coating, load vancomycin Ti coating could prevent the penetration hole structure caused by MRSA infection of the implant. The coating under the condition of MRSA pathogenic bacteria did not affect bone integration. Therefore, the vancomycin load on the pores structure of the Ti coating modification of orthopedic implants is expected to be used for the prevention and treatment of orthopedic-related infections implants.

\section{Authors' contributions}

Each Author has contributed substantially to the research. All authors read and approved the final manuscript.

\section{Authors' information}

Hao Zhang, Guang-Chao Wang and Peizhao Liu are co-first authors; Fang Ji and Hao Tang are co-corresponding authors.

\section{Availability of data and materials}

The data is deposited in the Department of Orthopedics of Changhai Hospital. Please contact the correspondence author Fang Ji, doctorjif@126.com, for the usage of data. 


\section{Ethics approval and consent to participate}

The subjects all gave their written informed consent in accordance with the Ethics Committee of Changhai Hospital.

\section{Conflicts of interest}

The authors declare that they have no competing interests.

\section{Acknowledgements}

This work was supported by the grants from Shanghai Committee of Science and Technology Foundation (No. 13411951500).

\section{References}

1 L. A. Cordova, V. Stresing, B. Gobin, P. Rosset, N. Passuti, F. Gouin, V. Trichet, P. Layrolle and D. Heymann, Clin. Sci., 2014, 127, 277-293.

2 J. Cobo and J. L. Del Pozo, Expert Rev. Anti-Infect. Ther., 2011, 9, 787-802.

3 H. O. Gbejuade, A. M. Lovering and J. C. Webb, Acta Orthop., 2015, 86, 147-158.

4 L. Zhao, P. K. Chu, Y. Zhang and Z. Wu, J. Biomed. Mater. Res., Part B, 2009, 91, 470-480.

5 B. Nie, T. Long, H. Ao, J. Zhou, T. Tang and B. Yue, Antimicrob. Agents Chemother., 2017, 61(1), e01766.

6 B. Nie, H. Ao, J. Zhou, T. Tang and B. Yue, Colloids Surf., B, 2016, 145, 728-739.

7 S. B. Goodman, Z. Yao, M. Keeney and F. Yang, Biomaterials, 2013, 34, 3174-3183.

8 B. e. Nie, H. Ao, C. Chen, K. Xie, J. Zhou, T. Long, T. Tang and B. Yue, $R S C$ Adv., 2016, 6, 46733-46743.

9 M. Godoy-Gallardo, C. Mas-Moruno, M. C. FernándezCalderón, C. Pérez-Giraldo, J. M. Manero, F. Albericio, F. J. Gil and D. Rodríguez, Acta Biomater., 2014, 10, 35223534 .
10 B. Jose, V. Antoci Jr, A. R. Zeiger, E. Wickstrom and N. J. Hickok, Chem. Biol., 2005, 12, 1041-1048.

11 H. Zhang, S. Yu, A. Tian, X. X. Xiang, W. Lin, A. Alquhali and X. Bai, Int. J. Nanomed., 2013, 8, 4379.

12 L. Zhang, J. Yan, Z. Yin, C. Tang, Y. Guo, D. Li, B. Wei, Y. Xu, Q. Gu and L. Wang, Int. J. Nanomed., 2014, 9, 3027.

13 Y. Roger, L. M. Schäck, A. Koroleva, S. Noack, K. Kurselis, C. Krettek, B. Chichkov, T. Lenarz, A. Warnecke and A. Hoffmann, Colloids Surf., B, 2016, 148, 104-115.

14 Q. Luo, Y. Huang, G. Zha, Y. Chen, X. Deng, K. Zhang, W. Zhu, S. Zhao and X. Li, J. Mater. Chem. B, 2015, 3, 784795.

15 Y. Huang, G. Zha, Q. Luo, J. Zhang, F. Zhang, X. Li, S. Zhao, W. Zhu and X. Li, Sci. Rep., 2014, 4, 6172.

16 G. C. Wang, H. Zhang, Q. Y. He, D. K. Tong, C. Ding, P. Z. Liu, Z. Q. Zhang, Y. T. Xie and F. Ji, RSC Adv., 2017, 7, 1956519575.

17 H. L. Tan, H. Y. Ao, R. Ma, W. T. Lin and T. T. Tang, Antimicrob. Agents Chemother., 2014, 58, 6016-6023.

18 B. Nie, H. Ao, T. Long, J. Zhou, T. Tang and B. Yue, Colloids Surf., B, 2017, 150, 183-191.

19 B. Thomes, P. Murray and D. Bouchier-Hayes, J. Bone Jt. Surg., Br. Vol., 2002, 84, 758-760.

20 D. Campoccia, L. Montanaro, P. Speziale and C. R. Arciola, Biomaterials, 2010, 31, 6363-6377.

21 P. Anguita-Alonso, A. D. Hanssen, D. R. Osmon, A. Trampuz, J. M. Steckelberg and R. Patel, Clin. Orthop. Relat. Res., 2005, 439, 43-47.

22 A. Chokr, D. Watier, H. Eleaume, B. Pangon, J. C. Ghnassia, D. Mack and S. Jabbouri, Int. J. Med. Microbiol., 2006, 296, 381-388.

23 J. Begun, J. M. Gaiani, H. Rohde, D. Mack, S. B. Calderwood, F. M. Ausubel and C. D. Sifri, PLoS Pathog., 2007, 3, e57.

24 P. S. Stewart and J. W. Costerton, Lancet, 2001, 358, 135.

25 H. L. Tan, W. T. Lin and T. T. Tang, Int. J. Artif. Organs, 2012, 35, 832-839.

26 L. Lazzarini, J. T. Mader and J. H. Calhoun, J. Bone Jt. Surg., Am. Vol., 2004, 86-A, 2305. 